\title{
L2 Learner Attention, Uptake, Memory and Affect in Corrective Feedback
}

\section{Liqin $\mathrm{Wu}$}

School of Languages and Media, Anhui University of Finance and Economics, Bengbu, China

Email: lilywulq@126.com

How to cite this paper: Wu, L. Q. (2021). L2 Learner Attention, Uptake, Memory and Affect in Corrective Feedback. Open Journal of Modern Linguistics, 11, 786-807. https://doi.org/10.4236/ojml.2021.115061

Received: September 2, 2021

Accepted: September 27, 2021

Published: September 30, 2021

Copyright (c) 2021 by author(s) and Scientific Research Publishing Inc. This work is licensed under the Creative Commons Attribution International License (CC BY 4.0).

http://creativecommons.org/licenses/by/4.0/ (c) (i) Open Access

\begin{abstract}
Quite different from the previous research on corrective feedback (CF), this paper is to explore learner cognitive psychology of CF in attention, uptake, memory and affect during the process of second language acquisition (SLA). An anonymous questionnaire survey of 1773 learners in four universities of Anhui province in China and 15 interviews with the participants in the survey in Anhui University of Finance and Economics (AUFE) reveal that the $\mathrm{CF}$ types most attracting a learner attention are elicitation and repetition in oral $\mathrm{CF}$ and collective $\mathrm{CF}$ in class and underlining the errors in written $\mathrm{CF}$; that the combination of the target language and the mother tongue is the most conducive to CF uptake and memory; that learners can understand the $\mathrm{CF}$ once they notice and are aware of it; looking the CF over several times contributes to memory; that most learners have initiatives of learning, and euphemistic CF and correcting alone after class are the least harmful to learner affect; that a learner cognitive psychology of CF is influenced by the thinking ways and values of his or her culture; that the individual differences in learner attention, uptake, memory and affect of CF and the different levels of second language proficiency are also the key factors that have to be considered when providing $\mathrm{CF}$. These findings have implications for teachers to catch learners' attention, make them understand, memorize and deploy $\mathrm{CF}$ correctly in L2 learning without hurting their affect.
\end{abstract}

\section{Keywords}

Attention, Uptake, Memory, Affect, CF

\section{Introduction}

Cognitive psychology is a complicated but omnipresent topic for teachers and learners, and plays a dominant role in L2 language teaching and learning. The bulk of research on L2 learner cognitive psychology has been carried out from 
perspectives of attention (Arnold, 2011; Polio, 2012; Robinson, 1995; Schmidt, 1995; Shintani \& Ellis, 2015), information-processing (McLaughlin et al., 1983; Smart, 2011), uptake (Krashen, 1982; Storch \& Wigglesworth, 2010), memory (Baddeley, 2003; Goo, 2012; Miyake \& Shah, 1999), and affect (Brown, 2001; Gregg, 1984; Krashen, 1985).

Teacher or learner cognition in language teaching or learning influences what teachers or learners do in the language teaching classroom (Borg, 2017; Rotjanawongchai, 2019; Wu et al., 2021). As an integral part of cognition, "the affective component contributes at least as much and often more to language learning than the cognitive skills" (Stern, 1983: p. 386), such affect as ego boundaries and attainments (Baran-€ucarz, 2012), motivation to learn languages in a multicultural world (Ushioda \& Dörnye, 2017), psychological constructs and behaviors (Scovel, 1978), personality (Coker \& Mihai, 2017), language stress and anxiety (Hashemi, 2011), self-confidence (Edwads \& Roger, 2015), self-esteem and self-concept (Habrat, 2013), and cognitive learning styles (Srichanyachon, 2011).

Therefore, the interdisciplinary research on applied linguistics and cognitive psychology contributes a lot to SLA.

\section{Attention, Uptake, Processing, Memory and Affect in CF}

Attention, uptake, processing, memory and affect are vital factors of learner cognitive psychology of CF that are closely connected and inseparable from one another. The factors may influence each other and produce different learning effects.

\subsection{Attention \& Uptake}

Schmidt (1995) argues that the extent of attention called noticing is an essential factor for learning L2 grammatical forms. Writing revision according to CF may result in pushed output and is conducive to noticing the grammatical forms that may otherwise be ignored and promoting storage of the features in memory (Arnold, 2011). Robinson's (1995) findings indicate that the differences in the consciously regulated processing demands of training tasks that result in differential performance on implicit and explicit learning and memory, and that the attentional demands and capacity and their individual differences influence the degree of noticing and SLA.

Learners can notice corrections if they are requested to or take time to look over the CF and carefully examine the errors (Polio, 2012; Shintani \& Ellis, 2015). But noticing an error does not mean being able to correct it (Guénette, 2012) since noticing and understanding are two different levels of awareness with the former at the lower level, the latter at the higher level where learners can draw inferences from one example, avoid the mistakes and express themselves correctly thereafter.

Research shows that linguistic and affective factors are influential in a learner uptake (and retention) (Storch \& Wigglesworth, 2010). When investigating 
young Japanese learners' incidental acquisition of English plural -s, Shintani \& Ellis (2010) find that comprehension-based instruction indicates an advantage for the complete beginners. "Comprehension is a necessary condition for language acquisition but is not sufficient" (Krashen, 1982: p. 66). The identical comprehensible input does not mean equal success for all L2 learners, something more than comprehensible input is needed, like motivation, self-confidence, etc. Emotional experience renders peptide hormonal substances releasing rapidly into the bloodstream, bringing about highly dramatic changes in our brain functions and our body state, which can either facilitate or impede learning (Arnold, 2011).

\subsection{Processing \& Memory}

The knowledge that learners obtain must be processed before it becomes digested. The information processing model in SLA highlights a progression through learning stages (McLaughlin et al., 1983). Information processing is influenced by the extent of attention that is affected by affect, and by the capability of processing the information that is affected by individual differences. Automatic processes differ from controlled ones where the latter pave the way for the former. This model was proved to be effective in the experiment of Hulstijin and Hulstijin (1984). They assume that learners are dissimilar either on the executive control dimension or on the metacognitive dimension, i.e. they differ between controlled and automatic processing, and between implicit and explicit knowledge of language. They argue that practising control should take time; making learners with a limited L2 capacity speak fast is to increase their errors. Content-centered message instead of the form-centered message is also to increase errors due to the processing channel overload.

Memory is often associated with working memory, a term coined by Pribram Miller, and Galanter in the 1960s to compare the mind to a computer (Baddeley, 2003). It is often thought of as a concept like short-term memory (STM) (Anderson, 1990: p. 150). As a cognitive resource, working memory “1) can be allocated to enable the maintenance and processing of information, 2) is inherently limited, and 3) differs in supply across individuals" (Miyake \& Shah, 1999: p. $136)$; is conceived as "the ability to maintain information in an active and readily accessible state, while concurrently and selectively processing new information" (Conway et al., 2007). It essentially amounts to "the contents of STM plus the limited-capacity controlled attention processes associated with the central executive that can be used to maintain some set of those STM units as the focus of attention" (Engle et al., 1999). It has been viewed as a critical cognitive construct in human cognition and has triggered diverse researches in cognitive psychology.

Ericsson and Kintsch (1995) maintain that long-term knowledge can be used to supplement the severely limited capacity of short-term working memory (ST-WM), and individual differences in long-term knowledge and skills may 
result in individual differences in working memory performance. Goo's (2012) study of CF and working memory capacity (WMC) suggests that individual differences in WMC significantly predicted, and mediated the effects of, recasts but not metalinguistic feedback, on the acquisition of the that-trace filter. That suggests that executive attention or attention control (a critical component of WMC) is involved in the noticing of recasts, but not in the noticing of metalinguistic feedback. In Mackey and colleagues' (2002) research on the role of WMC in interaction, noticing, and L2 English question development, it was found that more noticing (of recasts) occurred among high-span learners, whereas less noticing was associated with low-span learners.

\subsection{Affect}

The well-known Affective Filter Hypothesis suggests that variation between learners in the mental block, i.e. the affective filter hinders acquirers from fully utilizing the comprehensible input they receive for language acquisition. This affective filter might result from the acquirer's unmotivated behavior, lacking in self-confidence, or anxiousness (Krashen, 1985: p. 3).

The Affective Principles (Brown, 2001: pp. 22-26) consist of four principles: language ego, self-confidence, risk-taking and the language-culture connection. Firstly, the language ego, a learner's second identity, is a new mode of thinking, feeling, and acting that a learner develops as he learns to use a second language. When "intertwined with" L2, the language ego can easily create within the learner a sense of fragility, a defensiveness and a raising of inhibitions. Next, learners' belief in their capability of accomplishing the task is a partial factor for their eventual success. Therefore, learners' self-confidence or self-assessment is crucial to the successful learning. Thirdly, the successful language learners must be willing to become "gamblers" in the language game to produce and interpret language a bit beyond their absolute certainty. Lastly, the acculturation, social distance, and psychological adjustment are factors that have to be considered in SLA. It has been repetitively proven that successful SLA is closely relevant to social, cognitive, psychological, and affective factors and the role of affective variables in SLA is crucial to our understanding of the complexities of SLA (Young, 2013).

Different from the previous research on corrective feedback (CF), this research is to explore learner cognitive psychology of $\mathrm{CF}$ in attention, uptake, memory and affect during the process of SLA since it is difficult to obtain the processing data.

It attempts to answer the following questions:

1) In oral and written CF, which type (s) of CF most attract(s) a learner attention?

2) In oral and written $C F$, how many learners can understand, memorize and use the CF correctly?

3) And which type (s) of CF is (are) the least harmful to learner affect? 
The hypotheses are proposed as follows:

$\mathrm{H}_{1}$ : Indirect $\mathrm{CF}$ can catch a learner attention and will not hurt his or her affect since all the participants are Chinese.

$\mathrm{H}_{2}$ : A great majority of learners can understand, memorize and use the CF correctly.

\section{Method}

The research is comprised of a questionnaire survey with 41 items and 15 interviews to probe into L2 learner cognitive psychology of $\mathrm{CF}$ and the reasons behind the choices.

\subsection{The Questionnaire Survey}

The anonymous questionnaire survey used 5-point Likert scale to test L2 learner attention, uptake, memory and affect of CF. The survey was distributed, collected and calculated through an on-line questionnaire survey software. Two steps are taken to accomplish the survey.

\subsubsection{Preparation}

Before the questionnaire survey, the researchers conducted a pilot study in which there were 43 items on L2 learner attention, uptake, memory and affect and 54 students participated in it. The results of the pilot study indicated that the reliability value $(0.884)$ was satisfactory, while the validity value was below 0.5 . Then two items whose validity values were too low were deleted, and the others were revised.

\subsubsection{A Questionnaire Survey on a Large Scale}

In this questionnaire survey, there were altogether 41 items concerning attention, uptake, memory and affect (see Appendix) of oral or written CF. There were positive statements including double negations like not make a mistake and not hurt (items $1-7,9-10,12-16,20-22,24,28-30,33-36,38-40$ ) and negative statements including negative words like forget, make a mistake and hurt (items 8, 11, 17 - 19, 23, 25 - 27, 31 - 32, 37, 41). Items 1 - 15: the types of $\mathrm{CF}^{1}$ attracting the learner attention; items $16-25$ : the types of $\mathrm{CF}$ making the learner understand; items 26 - 34: memory of CF in different situations; items 35 - 41: affect of CF in different situations.

In each of the 41 items, there were five options: 1) It is never true of me. 2) It is occasionally true of me. 3) It is sometimes true of me. 4) It is usually true of me. 5) It is always true of me. Participants were required to select one of the five choices that best suits them.

\footnotetext{
${ }^{1}$ There are six types of oral CF: explicit, recast, clarifying request, meta-lingual, elicitation and repetition (Lyster \& Ranta, 1997; Lyster et al., 2013). In written CF, there are two types of CF: direct and indirect. Indirect includes marking the error, meta-lingual description, collective CF in class, providing the website and recast (Chandler, 2003; Ellis, 2009). In general, explicit or direct and implicit or indirect are the two major types of error correction applicable to both oral and written CF even though oral CF differs in method from the written one (Lyster et al., 2013). In this paper, when to correct, who is to correct and how to correct are classified into the types of written CF.
} 


\subsubsection{Participants}

The participants amounted to 1773 students in four universities in Anhui province. For gender, 743 (41.91\%) male, 1030 (58.09\%) female. Major: 22 (1.24\%) business English majors; 170 (9.59\%) English majors, 2 (0.11\%) art majors, and 1579 (89.05\%) other majors. Year: 1122 (63.28\%) freshmen, 221 (12.46\%) sophomores, $322(18.16 \%)$ juniors, 7 (0.39\%) seniors, 92 (5.19\%) first year postgraduates, and $9(0.51 \%)$ second year postgraduates.

\subsection{Interviews}

After obtaining the results of the anonymous questionnaire survey, random interviews were conducted among 15 participants ( 7 males, 8 females) in four schools of AUFE to know the deep reasons behind the choices. The questions in the interviews were the same as those in the questionnaire survey.

\section{Results, Analysis \& Discussion}

Results, analysis and discussion about an anonymous questionnaire survey and random interviews are provided here.

\subsection{The Questionnaire Survey}

The statistical data indicate that this survey is valid and reliable for its KMO is 0.942 , Cronbach $\alpha$ is 0.93 . The results are as follows (see Table 1 ).

As Table 1 indicates that all the choices are scattered in the five options with different percentages. But to find out the type of CF most attracting learners' attention, making them understand, and the situations for memory and affect, the researcher focuses on the sum of the option percentages of 3,4 , and 5 for the positive statements, and 3, 2 and 1 for the negative statements.

\section{Attention}

In oral $\mathrm{CF}$, the percentages of $\mathrm{CF}$ types that can catch a learner attention and make him or her aware of the errors are: explicit CF (64.643\%); repetition (66.49\%); clarification request (59.22\%); recast (66.33\%); meta-lingual CF (70.5\%); elicitation (70.9\%); criticism (57.65\%). As can be seen, elicitation most attract a learner attention.

Table 1. A questionnaire survey on L2 learner attention, uptake, memory and affect of CF.

\begin{tabular}{|c|c|c|c|c|c|c|}
\hline \multirow{3}{*}{ Item No } & \multicolumn{5}{|c|}{ Options \& Number of participants (\%) } & \multirow{3}{*}{ Total No (\%) } \\
\hline & 1 & 2 & 3 & 4 & 5 & \\
\hline & No (\%) & No (\%) & No (\%) & No (\%) & No (\%) & \\
\hline 1 & $188(10.6)$ & $439(24.76)$ & $520(29.33)$ & $446(25.16)$ & $180(10.15)$ & \\
\hline 2 & $174(9.81)$ & $420(23.69)$ & $539(30.4)$ & $469(26.45)$ & $171(9.64)$ & \\
\hline 3 & $235(13.25)$ & $488(27.52)$ & $554(31.25)$ & $377(21.26)$ & $119(6.71)$ & $1773(100)$ \\
\hline 4 & $169(9.53)$ & $428(24.14)$ & $573(32.32)$ & $432(24.37)$ & $171(9.64)$ & \\
\hline 5 & $124(6.99)$ & $399(22.5)$ & $662(37.34)$ & $442(24.93)$ & $146(8.23)$ & \\
\hline
\end{tabular}




\section{Continued}

\begin{tabular}{|c|c|c|c|c|c|}
\hline 6 & $103(5.81)$ & 413 (23.29) & $653(36.83)$ & $454(25.61)$ & $150(8.46)$ \\
\hline 7 & $304(17.15)$ & $447(25.21)$ & $589(33.22)$ & $318(17.94)$ & 115 (6.49) \\
\hline 8 & $223(12.58)$ & $488(27.52)$ & $603(34.01)$ & $359(20.25)$ & $100(5.64)$ \\
\hline 9 & $53(2.99)$ & $330(18.61)$ & $603(34.01)$ & $600(33.84)$ & $187(10.55)$ \\
\hline 10 & $50(2.82)$ & $294(16.58)$ & $606(34.18)$ & $593(33.45)$ & $230(12.97)$ \\
\hline 11 & $193(10.89)$ & $491(27.69)$ & $579(32.66)$ & $380(21.43)$ & $130(7.33)$ \\
\hline 12 & $100(5.64)$ & $432(24.37)$ & $702(39.59)$ & $418(23.58)$ & $121(6.82)$ \\
\hline 13 & $129(7.28)$ & $430(24.25)$ & $714(40.27)$ & 397 (22.39) & $103(5.81)$ \\
\hline 14 & $94(5.3)$ & 397 (22.39) & $688(38.8)$ & $467(26.34)$ & $127(7.16)$ \\
\hline 15 & $72(4.06)$ & 303 (17.09) & $562(31.7)$ & $500(28.2)$ & 336 (18.95) \\
\hline 16 & $47(2.65)$ & 303 (17.09) & $663(37.39)$ & $586(33.05)$ & $174(9.81)$ \\
\hline 17 & $84(4.74)$ & $383(21.6)$ & $738(41.62)$ & $435(24.53)$ & $133(7.5)$ \\
\hline 18 & $70(3.95)$ & $368(20.76)$ & $745(42.02)$ & $448(25.27)$ & $142(8.01)$ \\
\hline 19 & $71(4)$ & $334(18.84)$ & $628(35.42)$ & $541(30.51)$ & $199(11.22)$ \\
\hline 20 & $46(2.59)$ & $283(15.96)$ & $587(33.11)$ & $605(34.12)$ & $252(14.21)$ \\
\hline 21 & $54(3.05)$ & 345 (19.46) & $761(42.92)$ & $483(27.24)$ & $130(7.33)$ \\
\hline 22 & $82(4.62)$ & $379(21.38)$ & 809 (45.63) & $404(22.79)$ & $99(5.58)$ \\
\hline 23 & $161(9.08)$ & $451(25.44)$ & $722(40.72)$ & $355(20.02)$ & $84(4.74)$ \\
\hline 24 & $50(2.82)$ & $322(18.16)$ & $803(45.29)$ & $507(28.6)$ & $91(5.13)$ \\
\hline 25 & $152(8.57)$ & $550(31.02)$ & $750(42.3)$ & $257(14.5)$ & $64(3.61)$ \\
\hline 26 & $70(3.95)$ & $402(22.67)$ & $745(42.02)$ & $446(25.16)$ & $110(6.2)$ \\
\hline 27 & $60(3.38)$ & $343(19.35)$ & $689(38.86)$ & $515(29.05)$ & $166(9.36)$ \\
\hline 28 & $60(3.38)$ & 389 (21.94) & $765(43.15)$ & $440(24.82)$ & $119(6.71)$ \\
\hline 29 & $226(12.75)$ & $544(30.68)$ & $681(38.41)$ & $269(15.17)$ & $53(2.99)$ \\
\hline 30 & $91(5.13)$ & $427(24.08)$ & $747(42.13)$ & $422(23.8)$ & $86(4.85)$ \\
\hline 31 & $58(3.27)$ & $336(18.95)$ & $664(37.45)$ & $537(30.29)$ & $178(10.04)$ \\
\hline 32 & $171(9.64)$ & $517(29.16)$ & 707 (39.88) & $304(17.15)$ & $74(4.17)$ \\
\hline 33 & $64(3.61)$ & $431(24.31)$ & $802(45.23)$ & $391(22.05)$ & 85 (4.79) \\
\hline 34 & $208(11.73)$ & $556(31.36)$ & $689(38.86)$ & $270(15.23)$ & $50(2.82)$ \\
\hline 35 & $96(5.41)$ & $415(23.41)$ & $792(44.67)$ & $373(21.04)$ & $97(5.47)$ \\
\hline 36 & $52(2.93)$ & $261(14.72)$ & $651(36.72)$ & $517(29.16)$ & $292(16.47)$ \\
\hline 37 & $210(11.84)$ & $435(24.53)$ & $682(38.47)$ & $344(19.4)$ & $102(5.75)$ \\
\hline 38 & $106(5.98)$ & $368(20.76)$ & 693 (39.09) & $428(24.14)$ & $178(10.04)$ \\
\hline 39 & $51(2.88)$ & $240(13.54)$ & $596(33.62)$ & $562(31.7)$ & 324 (18.27) \\
\hline 40 & $59(3.33)$ & $279(15.74)$ & $603(34.01)$ & $513(28.93)$ & 319 (17.99) \\
\hline 41 & $210(11.84)$ & $477(26.9)$ & $663(37.39)$ & $295(16.64)$ & $128(7.22)$ \\
\hline
\end{tabular}


In written CF, the percentages of CF types that can NOT draw a learner attention and make him or her aware of the errors: marking the mistakes and letting the learner correct them (74.11\%); only providing the web link and letting the learners learn online and self-correct (71.24\%). The percentages of CF types that can catch learner attention and make them aware of their errors: underlining the errors and providing cues for errors (78.4\%); correcting the same or terrible blunders made by other students in class $(80.6 \%)$; indirect or implicit correction (69.99\%); self-correction (68.47\%); peer correction (72.3\%); correcting alone after class $(78.85 \%)$. Thus, correcting the same or serious blunders made by other students in class can most attract a learner attention.

Uptake

In oral CF, the percentages in various situations that can NOT make the learner understand the errors: using the incomprehensible words even after noticing and being aware of the errors (67.96\%); correcting only once $(66.73 \%)$; correcting quickly (58.26\%). The percentages in various situations that can make the learner understand the errors: after noticing and being aware of the errors $(80.25 \%)$; correcting the errors once in the target language and again in the mother tongue $(81.44 \%)$; timely correction contributing to understanding and correct application (77.49\%). Therefore, correcting the errors once in the target language and again in the mother tongue is the most conducive to CF uptake.

In written $\mathrm{CF}$, the percentages that can NOT make the learner understand the errors or use the language correctly: the delayed CF (75.24\%); even if having understood (81.89\%). The percentages that can make the learner understand the errors: understanding and correct application because the CF can be referred to anytime (74\%); understanding and correct application (79.02\%). Actually, as Guénette (2012) points out that understanding is at the higher level where learners can draw inferences from one example. If a learner really understands the CF, he or she can use it correctly. Therefore, it is inferred that superficial understanding of CF is not helpful to correct application.

\section{Memory}

In oral $\mathrm{CF}$, the percentages in various situations: remembering the errors when it is corrected, but soon forgetting it (68.64\%); remembering the errors when it is corrected, but forgetting it over time (61.59\%); remembering the CF only when the $\mathrm{CF}$ is given in the target language and again in the mother tongue (74.68\%); NOT repeating the same mistake for good memory (56.57\%); poor memory leading to the same mistake next time (71.34\%). Accordingly, 74.68\% of learners can memorize the CF only when the CF is given in the target language and again in the mother tongue.

In written $\mathrm{CF}$, the percentages in various situations: remembering the errors when it is corrected, but forgetting it over time (59.67\%); repeating the mistake for only focusing on the score or teacher's comments of the writing (78.68\%); remembering the error for having looked it over several times and NOT making the same mistake (72.07\%); remembering the CF for looking it over once and 
NOT making the same mistake (56.91\%). Hence, $72.07 \%$ of learners can remember the CF by looking it over several times.

Affect

Initiatives to learn $(73.49 \%)$; the percentages NOT hurting self-esteem, selfconfidence, or learning enthusiasm: self-correction (82.37\%); direct CF (73.27\%); euphemistic CF (83.59\%); correcting alone after class (80.93\%). The option percentages hurting self-esteem, self-confidence and learning enthusiasm: criticism (74.84\%); CF in front of the class (76.13\%). Thus, $73.49 \%$ of learners have initiatives of learning and euphemistic CF is the least harmful to learner affect.

Equally important, as shown in Table 1, all the choices by the participants in 41 items revolve around the options 2 (occasionally), 3 (sometimes) and 4 (usually) except items 7, 15, 20, 36, 39 and 40 which contains extreme choices 1 (never) or 5 (always). Among the options 2, 3 and 4, option 3 ranks first in percentage, which is the most favorite choice chosen by $29.23 \%-45.63 \%$ participants except item 20 whose choice is $4(34.12 \%)$ and whose option percentage is 1.01 more than that of $3(33.11 \%)$. The thinking ways and values of the Chinese people-the Golden Mean in our culture might account for the reason. In China, moderation is cherished, and people's behavior is not expected to go extremes. When happiness, anger, sorrow and joy are not expressed, they are in the state of the Mean. When they are expressed moderately, they are in the state of Harmony. The Mean is the most fundamental thing in the world, and harmony is a law followed by the whole world. When the Mean and Harmony are achieved, the heavy and the earth will be in their place, and all the things will grow and develop.

Besides the middle options, the extreme choices in items 7, 15, 20, 36, 39 and 40 also draw the researcher attention since their percentages are over 14. For $17.155 \%$ of learners, criticizing can never be helpful in catching their attention and make them aware of their mistake. For $18.95 \%$ of learners, correcting alone after class can always help them catch their attention and make them aware of their mistake. For $14.21 \%$ of learners, mother tongue always aids them to learn a foreign language well. For $16.47 \%$ and $18.27 \%$ of learners, self-correction and euphemistic CF can always respectively save learner face and prevent them from damaging their self-esteem, self-confidence or learning enthusiasm. For $17.99 \%$ of learners, correcting alone after class will always keep their self-esteem, self-confidence and learning enthusiasm. These findings echo the early research findings by Brown (2001), Goo (2012), Guénette (2012), Krashen (1982), Miyake \& Shah (1999) and Robinson (1995).

Therefore, realizing individual differences plays a vital role in foreign language teaching. Teachers are supposed to avoid CF by criticism and try to provide CF alone after class or euphemistic CF or mother tongue if necessary, and practice self-correction for those who have reached the expected level of language proficiency to satisfy learners with different needs. 


\subsection{Interviews}

As revealed in Table 2, the results of the 15 interviews may be different from those in the anonymous questionnaire survey. The probable reasons for these are diverse: the limited number of interviewees or majors or universities; face-to-face interviews are slightly different from an anonymous questionnaire survey in that face-saving is an important issue that has to be taken into account, esp. in our Chinese culture. But the findings are as significant as those in the anonymous questionnaire survey.

Table 2. Interviews on L2 learner attention, uptake, memory and affect of CF.

\begin{tabular}{|c|c|c|c|c|c|c|}
\hline \multirow{3}{*}{ Item No } & \multicolumn{5}{|c|}{ Options \& Number of participants (\%) } & \multirow{3}{*}{ Total No (\%) } \\
\hline & 1 & 2 & 3 & 4 & 5 & \\
\hline & No (\%) & No (\%) & No (\%) & No (\%) & No $(\%)$ & \\
\hline 1 & & $1(6.67)$ & $1(6.66)$ & $6(40)$ & $7(46.67)$ & \\
\hline 2 & & & $3(20)$ & $9(60)$ & $3(20)$ & \\
\hline 3 & $3(20)$ & $4(26.66)$ & $4(26.67)$ & $3(20)$ & $1(6.67)$ & \\
\hline 4 & $4(26.67)$ & & $6(40)$ & $3(20)$ & $2(13.33)$ & \\
\hline 5 & $2(13.33)$ & & $5(33.33)$ & $5(33.33)$ & $3(20)$ & \\
\hline 6 & $2(13.33)$ & $4(26.67)$ & $2(13.33)$ & $3(20)$ & $4(26.67)$ & \\
\hline 7 & $3(20)$ & $2(13.33)$ & $1(6.67)$ & $4(26.67)$ & $5(33.33)$ & \\
\hline 8 & $1(6.67)$ & $2(13.33)$ & $5(33.33)$ & $3(20)$ & $4(26.67)$ & \\
\hline 9 & & $1(6.67)$ & $2(13.33)$ & $7(46.67)$ & $5(33.33)$ & \\
\hline 10 & $1(6.67)$ & & & $6(40)$ & $8(53.33)$ & \\
\hline 11 & $4(26.67)$ & & $2(13.33)$ & $4(26.67)$ & $5(33.33)$ & \\
\hline 12 & $4(26.67)$ & & $3(20)$ & $6(40)$ & $2(13.33)$ & \\
\hline 13 & $2(13.33)$ & $5(33.33)$ & $3(20)$ & & $5(33.33)$ & \\
\hline 14 & $2(13.33)$ & $3(20)$ & $4(26.67)$ & $4(26.67)$ & $2(13.33)$ & \\
\hline 15 & & $2(13.33)$ & & $3(20)$ & $10(66.67)$ & \\
\hline 16 & & & $2(13.33)$ & $7(46.67)$ & $6(40)$ & \\
\hline 17 & & & $2(13.33)$ & $6(40)$ & $7(46.67)$ & \\
\hline 18 & $1(6.67)$ & $1(6.67)$ & $4(26.66)$ & $6(40)$ & $3(20)$ & \\
\hline 19 & & & $2(13.33)$ & $7(46.67)$ & $6(40)$ & \\
\hline 20 & $1(6.67)$ & $3(20)$ & $2(13.33)$ & $5(33.33)$ & $4(26.67)$ & \\
\hline 21 & & $5(33.33)$ & $3(20)$ & $6(40)$ & $1(6.67)$ & \\
\hline 22 & & $4(26.67)$ & $5(33.33)$ & $3(20)$ & $3(20)$ & \\
\hline 23 & $2(13.33)$ & $1(6.67)$ & $4(26.67)$ & $5(33.33)$ & $3(20)$ & $15(100)$ \\
\hline 24 & & & $6(40)$ & $6(40)$ & $3(20)$ & \\
\hline 25 & $3(20)$ & $3(20)$ & $8(53.33)$ & $1(6.67)$ & & \\
\hline 26 & $2(13.33)$ & $3(20)$ & $3(20)$ & $5(33.33)$ & $2(13.33)$ & \\
\hline 27 & $1(6.67)$ & $1(6.67)$ & $1(6.66)$ & $10(66.67)$ & $2(13.33)$ & \\
\hline 28 & & $3(20)$ & $5(33.33)$ & $7(46.67)$ & & \\
\hline 29 & $7(46.67)$ & $2(13.33)$ & $5(33.33)$ & $1(6.67)$ & & \\
\hline 30 & $1(6.67)$ & $2(13.33)$ & $2(13.33)$ & $8(53.33)$ & $2(13.33)$ & \\
\hline
\end{tabular}


Continued

\begin{tabular}{cccccc}
\hline 31 & & $2(13.33)$ & $4(26.67)$ & $8(53.33)$ & $1(6.67)$ \\
32 & $3(20)$ & $3(20)$ & $3(20)$ & $6(40)$ & \\
33 & $1(6.67)$ & $5(33.33)$ & $7(46.67)$ & $2(13.33)$ & \\
34 & $9(60)$ & $3(20)$ & $2(13.33)$ & & $1(6.67)$ \\
35 & $1(6.67)$ & $5(33.33)$ & $6(40)$ & $2(13.33)$ & $1(6.67)$ \\
36 & & $1(6.67)$ & $2(13.33)$ & $4(26.67)$ & $8(53.33)$ \\
37 & $6(40)$ & $4(26.67)$ & $1(6.67)$ & $4(26.66)$ & \\
38 & $1(6.67)$ & $2(13.33)$ & $2(13.33)$ & $4(26.67)$ & $6(40)$ \\
39 & & $2(13.33)$ & & $3(20)$ & $10(66.67)$ \\
40 & & & $1(6.67)$ & $5(33.33)$ & $9(60)$ \\
41 & $4(26.67)$ & $4(26.66)$ & $1(6.67)$ & $3(20)$ & $3(20)$ \\
\hline
\end{tabular}

\section{Attention}

In oral CF: 1. Those who chose 5 (7) and 4 (6) hold that explicit CF always or usually lets them know clearly where the error is and attract their attention and remember the error longer; One who chose 3 says that explicit CF enables her to immediately realize the grammar mistake. However, if the teacher keeps correcting in this way, she may feel a little embarrassed and lose her enthusiasm for learning. One who chose 2 says explicit CF is OK on a short-term basis, but in the long run it is better to teach the learner how to fish instead of giving them a fish. 2. Those who chose 5 (3) and 4 (9) report that teacher's adjusting the tone is always or usually a signal of an error that was used when they were in high school, and repetitions make them realize where the mistake is. But those who chose 3 (3) report teacher's adjusting the tone and repetition can attract their attention, but their awareness of errors depends on the difficulty level of the errors. 3. Those who chose 5 (1) and 4 (3) say that they are familiar with this kind of CF, which can always or usually attract their attention and awareness. Those who chose 3 (4), 2 (4) and 1 (3) prefer explicit CF to clarifying request because they say that clarifying request makes them more confused or nervous. They may know they are wrong, but don't know why. 4. Those who chose 5 (2) and 4 (3) always or usually like implicit CF and feel it more comfortable and relaxing. But those who chose 3 (6) and 1 (4) prefer explicit CF to implicit one, which makes them unable to be aware of where the error is. 5. Those who chose 5 (3) and 4 (5) hold that meta-lingual CF always or usually provides them an opportunity to have self-reflection. But those who chose 3 (5) and 1 (2) prefer direct $\mathrm{CF}$, for without which it is very hard for them to know the errors. 6. Those who chose 5 (4) and 4 (3) report that elicitation is always or usually good and impressive for them to find the errors independently. But those who chose 3 (2), 2 (4) and 1 (2) prefer direct CF. Otherwise they will not be aware of their errors and keep making the same mistakes. 7. Those who chose 5 (5) and 4 (4) think it is always or usually acceptable to be criticized for the errors for it can leave them a 
deep impression and know where the error is. Those who chose 3 (1), 2 (2) and 1 (3) dislike this kind of CF and think that it hurts self-esteem and is unable to help them be aware of their errors.

In Written CF: 8. Those who chose 5 (4), 4 (3) and 3 (5) hold that marking the mistakes can always or usually or sometimes not make learners aware of the errors. Moreover, they don't know how to correct them. Those who chose 2 (2) and 1 (1) think it is a good and impressive way to correct the errors. 9. Those who chose 5 (5) and 4 (7) believe that underlining the errors can attract their attention and they can find out the errors themselves. But those who chose 3 (2) and 2 (1) are unable to correct the errors themselves even if they know they are wrong. 10. Those who chose 5 (8) and 4 (6) think that collective CF in class can always or usually draw their attention and make them aware of the error because they say that it is very likely for them to make such a mistake now and then. But only one interviewee says that she never makes the similar mistake, and this way of CF will mislead her. 11. Those who chose 5 (5) and 4 (4) report that they may not have time to have access to the website. And some say once they surf the internet, they may not go to the website provided by the teacher, but will either go to other websites for reference for learners of high level of proficiency or play computer games for those who are addicted to computer games. Those who chose 3 (2) and 1 (4) think it is sometimes or never the case. But they insist that the teacher's provision of correct answers is better than the website for in that case they can understand the CF fully. 12. Those who chose 5 (2) and 4 (6) think that indirect or implicit CF can always or usually attract their attention and awareness of the errors and are as impressive as direct or explicit CF. But those who chose 3 (3) and 1 (4) prefer direct or explicit CF, and they hold that indirect or implicit CF cannot attract their attention and they are not clear what the teacher intends to say, let alone be aware of their own errors. 13. Those who chose 5 (5) report that they are always or usually accustomed to self-correction and have been practicing it. Those who chose 3 (3), 2 (5) and 1 (2) report that they are poor in their language levels and weak in their awareness of self-correction. Consequently, this kind of CF can sometimes, occasionally or never attract their attention and or arouse their awareness of the errors. 14. Those who chose 5 (2) and 4 (4) think that peer CF is always or usually suitable and acceptable since they have experienced it. Those who chose 3 (4), 2 (3) and 1 (2) report that the quality of peer CF is constrained by the peer's level of the second language. They believe that in most cases peers with the same level of language proficiency cannot find the errors, either. 15. Those who chose 5 (10) and 4 (3) report correcting alone after class is always or usually impressive and memorable. Those who chose 2 (2) dislike this way of correction for they think it will take up their leisure time for other activities.

\section{Uptake}

In oral CF: 16. Those who chose 5 (6) and 4 (7) believe that after noticing and being aware of the errors, they can always or usually understand where the error 
is. But those who chose 3 (2) feels that sometimes they can understand, sometimes they cannot. 17. Those who chose 5 (7) and 4 (6) always or usually cannot understand the error even after noticing and being aware of it if the teacher uses incomprehensible words. But those who chose 3 (2) think that sometimes it is so. 18. Those who chose 5 (3) and 4 (6) think that they can always or usually not understand or memorize what the teacher says if he or she only says it once. Those who chose 3 (4), 2 (1) and 1 (1) think that it is sometimes or occasionally or never the case. 19. Those who chose 5 (6) and 4 (7) say that if the CF is provided quickly, they always or usually have no time to digest, and cannot remember or understand what the teacher says. Those who chose 3 (2) think sometimes it is so. 20. Those who chose 5 (4) and 4 (5) hold that mother tongue is helpful to their error understanding. But those who chose 3 (2), 2 (3) and 1 (1) don't think it is necessary to repeat the error in their mother tongue for it will distract their attention from the target language to the mother tongue and do bad to second language learning. 21. Those who chose 5 (1) and 4 (6) think that they can always or usually understand the errors and use the language correctly for the oral CF is in time. Those who chose 3 (3) and 2 (5) report that they can sometimes or occasionally understand the errors or use the language correctly for they have few opportunities to speak foreign language and pay little attention to oral English. They think it requires practice to learn spoken foreign language well.

In written CF: 22. Those who chose 5 (3) and 4 (3) think that they can always or usually understand the CF and use it correctly later because it very convenient for them to go back to the written correction by the teacher. But those who chose 3 (5) and 2 (4) sometimes have no time to refer to the CF or forget the CF. 23. Those who chose 5 (3) and 4 (5) believe that delay will always or usually make them unable to understand the errors and pay little attention CF. But those who chose 3 (4), 2 (1) and 1 (2) hold that it is sometimes or occasionally or never the case. And practice makes perfect. 24. Those who chose 5 (3) and 4 (6) can always or usually use the CF correctly next time. But those who chose 3 (6) don't believe they can use it correctly next time for sometimes they may forget the $\mathrm{CF}$ and sometimes the errors are difficult ones which are beyond their abilities. 25. One who chose 4 reports that usually even if he understands the errors, he still cannot use them correctly next time. Those who chose 3 (8) and 2 (3) think understanding sometimes or occasionally depends on the difficult level and memory of CF. Those who chose 1 (3) think it is never the case.

\section{Memory}

In oral CF: 26. Those who chose 5 (2) and 4 (5) report that oral CF is always or usually more likely to be forgotten than written CF. And if they do not review and consolidate the CF, they will soon forget it. But those who chose 3 (3), 2 (3) and 1 (2) report that they can remember the CF on a short-term basis. 27. Those who chose 5 (2) and 4 (10) report that they tend to forget the CF with the passage of time if they do not review the CF because of their short-term memory. One who chose 3 (1) report that sometimes it is the case. Those who chose 2 (1) 
and 1 (1) believe that if they really memorize the CF, they will not forget it. 28. Those who chose 4 (7) think the use of mother tongue is usually a good way to help learner understand and memorize the mistake. Those who chose 3 (5), 2 (3) report that this cannot solve the problem of memory. It is unnecessary to correct the simple mistake again in the mother tongue and they can memorize it. 29. One who chose 4 reports that he can usually memorize most of the CF, but forgets a minor part of it. He will usually not make the same mistake again. Those who chose 3 (5) report sometimes they have a good memory on a short-term basis, they sometimes forget the CF. Those who chose 2 (2) and 1 (7) report a bad memory. 30. Those who chose 5 (2) and 4 (8) report that they always or usually tend to forget the CF and repeat the same mistake. But those who chose 3 (2) think it is sometimes so. Those who chose 2 (2) and 1 (1) can memorize the CF and will not repeat the mistake.

In written CF: 31 . Those who chose 5 (1) and 4 (8) say they always or usually remember the CF when it is corrected but if they do not review the $\mathrm{CF}$, they tend to forget it. It is sometimes or occasionally so for those who chose 3 (4) and 2 (2). 32. Those who chose 4 (6) think they usually repeat the error because they ignore the CF either for paying much attention to the score or for they posses writing templates that are more valuable than teacher's CF in their minds. Those who chose 3 (3) say that it is true for them sometimes. Those who chose 2 (3) and 1 (3) report that they think that score and CF are equally important to improve their writing ability. 33. Those who chose 4 (2) think looking over the CF several times is usually conducive to memory and avoiding the same errors. But those who chose 3 (7) hold that sometimes it rests with the difficulty level. Those who chose 2 (5) and 1 (1) report that they will make the same mistake next time because of their poor memory. 34. One who chose 5 can remember the error by looking it over once and will not make the same error next time. Those who chose 3 (2) say sometimes it is so. Those who chose 2 (3) and 1 (9) say that it is absolutely impossible for them to memorize the CF if they just look it over one time. They have to look at it many times before memorizing it.

Affect

One who chose 5 always self-corrects the errors. It is usually the case for 2 who chose 4 . It is sometimes the case for those who chose 3 (6) only when dealing with the tests. Those who chose 2 (5) and 1 (1) report they are lack of initiatives and self-correction is beyond their ability. 36. Those who chose 5 (8) and 4 (4) think self-correction will always or usually not hurt self-esteem, self-confidence or learning enthusiasm. Those who chose 3 (2) and 2 (1) admit self-correction is good, but sometimes or occasionally beyond their ability. 37 . Those who chose 4 (4) report they are usually in depressed spirit if being criticized. One who chose 3 says that criticism might trigger unsteady mood. However, those who chose 2 (4) and 1 (6) think this kind of CF is impressive and does good to their language learning. 38. Those who chose 5 (6) and 4 (4) says direct CF is always or usually helpful to correcting their errors. Those who 3 (2), 2 (2) and 1 (1) think it is 
sometimes or occasionally or never the case. 39. Those who chose 5 (10) and 4 (3) think euphemistic CF is always or usually acceptable and does not hurt self-respect, self -confidence or learning enthusiasm. But one of the two who chose 2 thinks that self-confidence is actually hurt where there is CF. The other prefers direct CF to euphemistic one. 40. Those who chose 5 (9) and 4 (5) report that correcting errors alone after class is always or usually a better way of providing $\mathrm{CF}$, impressive and helpful to learning. It does not hurt self-respect, self-confidence or learning enthusiasm. But one who chose 3 feels it painful. 41 . Those who chose 5 (3) and 4 (3) think CF in front of the class always or usually hurts their self-esteem, self-confidence and learning enthusiasm. One who chose 3 say it is sometimes the case. But those who chose 2 (4) and 1 (4) think it is occasionally or never the case. On the contrary, it is helpful to correcting the mistakes.

Briefly, the difficulty level of the errors and the individual differences in language proficiency are key factors that should be considered in attracting learner awareness of errors, uptake, memory and affect. It is better for the teacher to create a learning environment of foreign language. One has to be in the water before he learns to swim. And the fantastic development of the internet makes it possible for the learners to have an easy access to the knowledge outside the university. Most learners memorize CF only on a short-term basis. Revision of $\mathrm{CF}$ and practicing are necessary to understand, memorize and use the language correctly. Cultural factor is another aspect that should be taken into account in L2 learning. For example, in the traditional Chinese culture, respecting teachers, including taking teacher criticism seriously, is not only to respect for the sages, but also necessary for inheriting sages' knowledge. Saving learner face is needed in class, esp., in Chinese culture for those who are sensitive to any type of CF.

Meanwhile, to know the detailed statistical date of the interviews, the researcher takes the options 3, 4 and 5 in the positive statements but 3,2 and 1 in the negative statements as the reference values, the following results are obtained:

\section{Attention}

In oral $\mathrm{CF}$, the percentages that approve the type of $\mathrm{CF}$ role in their attention and awareness: explicit (93.33\%); repetition (100\%); clarifying request $(53.34 \%)$; recast $(73.33 \%)$; meta-lingual (86.66\%); elicitation (60\%); criticizing (66.67\%). Thus, repetition is the most popular way of attracting learner attention to error correction.

In written $\mathrm{CF}$, indirect $\mathrm{CF}$ by marking the errors (53.33\%); indirect CF by underlining the errors (93.33\%); indirect CF by collective error correction (93.33\%); indirect CF by providing the web link (40\%); indirect CF by implicit providing the correct forms (73.33\%); self-correction (53.33\%); peer correction (66.67\%); correction alone after class (86.67\%). As indicated, the CF types most attracting learner attention are indirect correction by underlining the errors and collective error correction. 


\section{Uptake}

In oral $\mathrm{CF}$, the percentages of $\mathrm{CF}$ situations in which the learner can NOT understand the CF: even after noticing and being aware of the error (13.33\%); saying once (40\%); saying quickly (13.33\%); the percentages of CF situations in which the learner can understand the CF: after noticing and being aware of the error (100\%); saying in target language and repetition in mother tongue (73.33\%); timely correction (66.67\%). Therefore, learners can understand the CF if they notice and are aware of it.

In written CF, the percentages that the learner can NOT understand or apply correctly the CF: delayed CF (46.67\%); incorrect application even if understanding (93.33\%). The percentages that the learner can understand and apply correctly the CF: the convenient retrospect (73.33\%); correct application after understanding (100\%). The percentage of incorrect application even if understanding seems to conflict with that of correct application after understanding. After carefully examining the results of the interviews, it is found that correct application of $C F$ is constrained by the difficulty level of the CF. If the difficulty level is high, the learners will not be able to apply it correctly or vice versa.

\section{Memory}

In oral CF, forgetting soon (53.33\%); forgetting over time (20\%); first in target language then in mother tongue (80\%); NOT making the same mistakes for good memory (40\%); making the same mistakes for poor memory (33.33\%). Thus, the combination target language and the mother tongue most contributes to memory.

In written CF: forgetting over time (40\%); focusing on the score or teacher's comments of the writing (60\%); NOT making the same mistakes for looking them over several times (60\%), NOT making the same mistakes for one-time revision in memory (20\%). Given that most learners have short-term memory, repetitive revision of CF strengthens memory.

Affect

$60 \%$ and $73.49 \%$ of learners learn on their own initiative. The situations that do NOT hurt self-esteem, self-confidence, or learning enthusiasm: self-correction (93.33\%); direct CF (80\%); euphemistic CF (86.66\%); correcting alone after class $(100 \%)$. The situations that hurt self-esteem, self-confidence, and learning enthusiasm: criticizing (73.34\%); CF in front the class (60\%). Thus, for $100 \%$ learners, correcting alone after class will not hurt self-esteem, self-confidence, and learning enthusiasm, which also mirrors the influence of the Chinese face-saving culture.

To sum up, all the results in the anonymous questionnaire survey and the interviews demonstrate that indirect error correction in both oral and written $\mathrm{CF}$ can catch a learner attention and will not hurt his or her affect, which conforms to $\mathrm{H}_{1}$. A great majority of learners can understand the CF once they notice and are aware of it and can memorize the $\mathrm{CF}$ when it is given in the target language and again in the mother tongue and looked over several times in oral CF and written CF respectively, which conditionally conforms to $\mathrm{H}_{2}$. 


\section{Conclusion}

This research focuses on attention, uptake, memory and affect of L2 cognitive psychology of CF and finds out some instructive clues for CF in SLA. These findings have implications for L2 teachers who decide which types of CF are most effective and adjust their ways of CF while keeping in mind learner individual differences in cognitive psychology, levels of language proficiency and cultural factors. $\mathrm{Bu}$ it is easier said than done. How to incorporate the results into the classroom and provide $\mathrm{CF}$ in a proper way to learners with different cognitive psychology, different levels of language proficiency and different cultural backgrounds is a great challenge for teachers so as to attract the learners' attention, make them really understand, memorize and deploy the CF correctly in their later life.

\section{Acknowledgement}

This research is funded by The Key Anhui Humanities and Social Sciences Planned Project Fund (AHSKZ2018D16).

\section{Conflicts of Interest}

The author declares no conflicts of interest regarding the publication of this paper.

\section{References}

Anderson, J. R. (1990). Cognitive Psychology and Its Implications. Freeman.

Arnold, J. (2011). Attention to Affect in Language Learning. Anglistik: International Journal of English Studies, 22, 11-22.

Baddeley, A. (2003). Working Memory: Looking Back and Looking Forward. Nature Reviews Neuroscience, 4, 829-839. https://doi.org/10.1038/nrn1201

Baran-Łucarz, M. (2012). Ego Boundaries and Attainments in FL Pronunciation. Studies in Second Language Learning and Teaching, 2, 45-66. https://doi.org/10.14746/ssllt.2012.2.1.3

Borg, S. (2017). Teacher Cognition in Language Teaching: A Review of Research on What Language Teachers Think, Know, Believe, and Do. Language Teaching, 36, 81-109. https://doi.org/10.1017/S0261444803001903

Brown, H. D. (2001). Teaching Principles: An Interactive Approach to Language Pedagogy. Foreign Language Teaching and Research Press.

Chandler, J. (2003). The Efficacy of Various Kinds of Error Feedback for Improvement in the Accuracy and Fluency of L2 Student Writing. Journal of Second Language Writing, 12, 267-296. https://doi.org/10.1016/S1060-3743(03)00038-9

Coker, C., \& Mihai, F. (2017). Personality Traits and Second Language Acquisition: The Influence of the Enneagram on Adult ESOL Students. TESOL Journal, 8, 432-449. https://doi.org/10.1002/tesj.281

Conway, A. R. A., Jarrold, C., Kane, M. J., Miyake, A., \& Towse, J. N. (2007). Variation in Working Memory: An Introduction. In A. R. A. Conway, C. Jarrold, M. J. Kane, A. Miyake, \& J. N. Towse (Eds.), Variation in Working Memory (pp. 3-17). Oxford Uni- 
versity Press. https://doi.org/10.1093/acprof:oso/9780195168648.003.0001

Edwads, E., \& Roger, P. S. (2015). Seeking out Challenges to Develop L2 Self-Confidence: A Language Learner's Journey to Proficiency. TESL-EJ, 18, 1-24.

Ellis, R. (2009). Corrective Feedback and Teacher Development. L2 Journal, 1, 3-18. https://doi.org/10.5070/L2.V1I1.9054

Engle, R. W., Tuholski, S. W., Laughlin, J. E., \& Conway, A. R. A. (1999). Working Memory, Short-Term Memory, and General Fluid Intelligence: A Latent Variable Approach. Journal of Experimental Psychology: General, 128, 309-331. https://doi.org/10.1037/0096-3445.128.3.309

Ericsson, K. A., \& Kintsch, W. (1995). Long-Term Working Memory. Psychological Review, 102, 211-245. https://doi.org/10.1037/0033-295X.102.2.211

Goo, J. (2012). Corrective Feedback and Working Memory Capacity in Interaction-Driven. Studies in Second Language Acquisition, 34, 445-474. https://doi.org/10.1017/S0272263112000149

Gregg, K. R. (1984). Krashen’s Monitor and Occam's Razar. Applied Linguistics, 5, 79-100. https://doi.org/10.1093/applin/5.2.79

Guénette, D. (2012). The Pedagogy of Error Correction: Surviving the Written Corrective Feedback Challenge. TESL Canada Journal/Revue du Canada, 30, 117-126. https://doi.org/10.18806/tesl.v30i1.1129

Habrat, A. (2013). The Effect of Affect on Learning: Self-Esteem and Self-Concept. In E. Piechurska-Kuciel, \& E. Szymańska-Czaplak (Eds.), Language in Cognition and Affect (pp. 239-253). Springer. https://doi.org/10.1007/978-3-642-35305-5 14

Hashemi, M. (2011). Language Stress and Anxiety among the English Language Learners. Procedia-Social and Behavioral Sciences, 30, 1811-1816. https://doi.org/10.1016/j.sbspro.2011.10.349

Hulstijin, J., \& Hulstijin, W. (1984). Grammatical Errors as Function of Processing Constraints and Explicit Knowledge. Language Learning, 43, 23-43. https://doi.org/10.1111/j.1467-1770.1984.tb00994.x

Krashen, S. (1982). Principles and Practice in Second Language Acquisition. Pergamon Press.

Krashen, S. (1985). The Input Hypothesis: Issues and Implication. Longman.

Lyster, R., \& Ranta, L. (1997). Corrective Feedback and Learner Uptake. Studies in Second Language Acquisition, 19, 37-66. https://doi.org/10.1017/S0272263197001034

Lyster, R., Saito, K., \& Sato, M. (2013). Oral Corrective Feedback in Second Language Classrooms. Language Teaching, 46, 1-40. https://doi.org/10.1017/S0261444812000365

Mackey, A., Philp, J., Fujii, A., Egi, T., \& Tatsumi, T. (2002). Individual Differences in Working Memory, Noticing of Interactional Feedback and L2 Development. In P. Robinson (Ed.), Individual Differences and Instructed Language Learning (pp. 181-208). John Benjamins Publishing Company. https://doi.org/10.1075/1llt.2.12mac

McLaughlin, B., Rossman, R., \& McLeod, B. (1983). Second Language Learning: An Information-Processing Perspective. Language Learning, 33, 135-158.

https://doi.org/10.1111/j.1467-1770.1983.tb00532.x

Miyake, A., \& Shah, P. (Eds.) (1999). Models of Working Memory: Mechanisms of Active Maintenance and Executive Control. Cambridge University Press. https://doi.org/10.1017/CBO9781139174909

Polio, C. (2012). The Relevance of Second Language Acquisition Theory to the Written 
Error Correction Debate. Journal of Second Language Writing, 21, 375-389. https://doi.org/10.1016/j.jslw.2012.09.004

Robinson, P. (1995). Attention, Memory, and the "Noticing" Hypothesis. Language Learning, 452, 283-331. https://doi.org/10.1111/j.1467-1770.1995.tb00441.x

Rotjanawongchai, S. (2019). Teacher Cognition of Corrective Feedback in the EFL Speaking Classroom: A Case Study of Thai University Teachers. PhD Thesis, University of York.

Schmidt, R. (1995). Consciousness and Foreign Language Learning: A Tutorial on the Role of Attention and Awareness in Learning. Attention and Awareness in Foreign Language Learning, 9, 1-64.

Scovel, T. (1978). The Effect of Affect on Foreign Language Learning: A Review of the Anxiety Research. Language Learning, 28, 129-142. https://doi.org/10.1111/j.1467-1770.1978.tb00309.x

Shintani, N., \& Ellis, R. (2010). The Incidental Acquisition of English Plural $-s$ by Japanese Children in Comprehension-Based and Production-Based Lessons. Studies in Second Language Acquisition, 32, 607-637. https://doi.org/10.1017/S0272263110000288

Shintani, N., \& Ellis, R. (2015). Does Language Analytical Ability Mediate the Effect of Written Feedback on Grammatical Accuracy in Second Language Writing? System, 49, 110-119. https://doi.org/10.1016/j.system.2015.01.006

Smart, J. J. C. (2011). The Mind/Brain Identity Theory. In E. N. Zalta (Ed.), The Stanford Encyclopedia of Philosophy. Stanford University.

Srichanyachon, N. (2011). Cognitive Learning Styles of EFL Students. Journal of College Teaching \& Learning (TLC), 8, 15-23. https://doi.org/10.19030/tlc.v8i2.3553

Stern, H. H. (1983). Fundamental Concepts of Language Teaching. Oxford University Press.

Storch, N., \& Wigglesworth, G. (2010). Learners' Processing, Uptake, and Retention of Corrective Feedback of Writing. Studies in Second Language Acquisition, 32, 303-334. https://doi.org/10.1017/S0272263109990532

Ushioda, E., \& Dörnye, Z. (2017). Beyond Global English: Motivation to Learn Languages in a Multicultural World: Introduction to the Special Issue. The Modern Language Journal, 101, 451-454. https://doi.org/10.1111/modl.12407

Wu, L. Q., Wu, Y., \& Zhang, X. Y. (2021). An On-Line Questionnaire Survey on Students' Views and Teachers' Practices in Corrective Feedback in Teaching Chinese to Speakers of Other Languages. International Linguistics Research, 4, 1-13. https://doi.org/10.30560/ilr.v4n1p1

Young, D. J. (2013). Affective Factors and Second Language Spanish. In K. L. Geeslin (Ed.), The Handbook of Spanish Second Language Acquisition (pp. 369-385). John Wiley \& Sons. https://doi.org/10.1002/9781118584347.ch21 


\section{Appendix}

\section{A Questionnaire Survey on Learner Attention, Uptake, Memory and Af- fect of CF}

This is an anonymous survey about L2 attention, uptake, memory and affect of CF. The data collected are not used for profits but for scientific research to improve the quality of language teaching and learning. In each of the following items, there are five options: 1) It is never true of me. 2) It is occasionally true of me. 3) It is sometimes true of me. 4) It is usually true of me. 5) It is always true of me. Please select one of the five choices that best suits you. The five choices in the following are omitted to save the length of the survey. ${ }^{\star} \mathrm{S}=$ student; $\mathrm{T}=$ teacher

\section{Attention}

Oral $C F$

1) Explicit error correction can catch my attention and make me aware of my errors.

S: I want to talk is ...

T: No. The correct way is what I want to talk is ... (explicit or direct)

2) That the teacher adjusts his/her tone (e.g., using rising tone) and repeats my errors can catch my attention and make me aware of the errors.

S: She go to school early in the morning.

$\mathrm{T}$ : She go to school? (repetition or indirect)

3) The expressions like "Excuse me?" or "I don't understand" can catch my attention and make me aware of my errors.

S: I like you jia (Correct Chinese: yu jia; English meaning: yoga)

T: "Excuse me?" (clarification request or indirect)

4) That the teacher implicitly corrects the errors or provides the correct expressions can catch my attention and make me aware of my errors.

S: I like Yogurt exercise in my spare time.

T: Good. You like Yoga exercise in your spare time. (recast or indirect)

5) That the teacher asks questions instead of providing the correct answer can catch my attention and make me aware of my errors. (meta-lingual CF or indirect)

S: It saves labors.

$\mathrm{T}$ : Do you think that labor can be used in plural form?

6) That the teacher elicits the correct form directly by asking me questions or guiding me to the correct expression can catch my attention and make me aware of my errors.

S: 我迟到了, 大大的对不起。(I am late. I am big big sorry.)

$\mathrm{T}$ : 我迟到了, 非常……? (I am late. I am terribly ...?)

S: 我迟到了, 非常对不起。(I am late. I am terribly sorry.) (elicitation or indirect)

7) Criticizing my errors can catch my attention and make me aware of my errors. 
S: John has bought a small car.

T: You have learned the grammar; how can you make such a blunder mistake? (criticism or indirect)

Written $C F$

8) That the teacher just marks the mistakes and lets me correct them can NOT catch my attention and make me aware of my errors. (Indirect)

9) That the teacher underlines the errors and writes "Can these two words go together?" can catch my attention and make me aware of the errors. (Indirect)

10) That the teacher corrects the same or serious blunders made by other students in class (collective CF) can catch my attention and make me aware of the errors. (Indirect)

11) That the teacher only provides the web link and lets the students learn online and self-correct can NOT catch my attention and make me aware of my errors. (Indirect)

12) That the teacher indirectly corrects the mistake or implicitly provides the correct expression instead of pointing out the mistake directly or explicitly can catch my attention and make me aware of my errors. (Indirect)

13) Self-correction can catch my attention and make me aware of my errors. (Self-correction)

14) Peer correction can catch my attention and make me aware of my errors. (Peer correction)

15) That the teacher corrects my errors alone after class can catch my attention and make me aware of my errors. (correction alone after class or indirect)

\section{Uptake}

Oral CF

16) After noticing and being aware of the errors, I can understand where the error is.

17) Even after noticing and being aware of the errors, I still can NOT understand what is wrong if the teacher uses the incomprehensible words.

18) If the teacher corrects the error only once, I still can NOT understand the mistakes.

19) If the teacher corrects the error quickly, I still can NOT understand the mistakes.

20) If the teacher provides the CF once in the target language and again in my mother tongue, I can understand the mistake.

21) I can understand the oral mistakes and use them correctly later because the mistakes are timely corrected.

Written $C F$

22) I can understand my writing mistakes and use them correctly later because I can always refer to corrections since they are always there.

23) I can NOT understand my writing mistakes or use them correctly later because the CF is delayed and the mistakes will be ignored.

24) When I understand my mistakes, I can use them correctly next time. 
25) Even if I understand my mistakes, I still can NOT use them correctly next time.

\section{Memory}

Oral CF

26) I remember the error when it is corrected, but soon forget it.

27) I remember the error when it is corrected, but forget it over time.

28) I can memorize the CF only on the condition that the teacher corrects the mistake in the target language, then again in the mother tongue.

29) I can remember the CF and will NOT make the same mistake again.

30) I often forget the CF and will make the same mistake again.

Written $C F$

31) I remember the CF when it is corrected, but I forget it over time.

32) I will repeat the mistake later for I only focus on the score or teacher's comments of the writing, ignoring $\mathrm{CF}$ when getting the $\mathrm{CF}$.

33) I will remember the CF if I look it over several times and will NOT make the same mistake.

34) I will remember the CF if I look it over once and will NOT make the same mistake.

Affect

35) I often self-correct my errors whether the teacher asks me or not, because I think learning is my own business.

36) Self-correction will NOT hurt my self-esteem, self-confidence, and learning enthusiasm.

37) Criticizing my errors will hurt my self-esteem, self-confidence and learning enthusiasm.

38) Direct CF will NOT hurt my self-esteem, self-confidence, and learning enthusiasm.

39) Euphemistic CF will NOT hurt my self-esteem, self-confidence, and learning enthusiasm.

40) Correcting my errors alone after class will NOT hurt my self-esteem, self-confidence, or learning enthusiasm.

41) CF in front of the class will hurt my self-esteem, self-confidence and learning enthusiasm. 